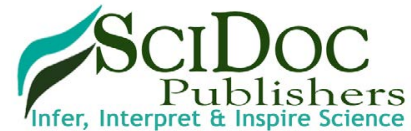

International Journal of Anesthesiology \& Research (IJAR)

ISSN 2332-2780

\title{
Sedoanalgesia Administration with Propofol and Ketamine for Minor Urologic Interventions
}

Research Article

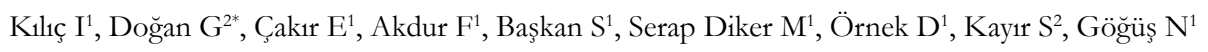

${ }^{1}$ Ankara Numune Training and Research Hospital, Anesthesiology and Reanimation Clinic, Ankara, Turkey.

${ }^{2}$ Hitit University Corum Education and Research Hospital, Anesthesiology and Reanimation Clinic, Çorum, Turkey.

\section{Abstract}

\begin{abstract}
Aim: In this study we aimed to administer sedoanalgesia with propofol and ketamine combination in patients undergoing planned minor urologic interventions with limited anesthesia. By combining these two medications, lower doses may be used, and we aimed to provide sufficient sedation, analgesia and amnesia without disrupting hemodynamic and respiratory stability and to increase patient and surgeon satisfaction.

Material-Method: The study included 53 patients with planned minor urologic interventions aged from 19 to 85 years and physical situation ASA I-III. After six hours starvation, patients were taken to the surgery. Patients were monitored for electrocardiography (ECG), oxygen saturation $\left(\mathrm{SPO}_{2}\right)$ and non-invasive blood pressure. For use if necessary a nasal $\mathrm{O}_{2}$ cannula was inserted. A vein in the back of the left hand was opened and $5 \mathrm{ml} / \mathrm{min}$ isotonic sodium chloride infusion was begun. Patient heart rate (HR), systolic arterial pressure (SAP), diastolic arterial pressure (DAP), mean arterial pressure (MAP) and oxygen saturation $\left(\mathrm{SpO}_{2}\right)$ values were measured and a 5 point sedation scale (Table 1) was used to measure sedation scores. Basal values were recorded $(0 \mathrm{~min})$. Later patients were randomly divided into two groups with Group I administered intraurethral lidocaine gel for local anesthesia by the surgeon, while Group II were administered intravenous $0.015 \mathrm{mg} / \mathrm{kg}$ midazolam, $0.5 \mathrm{mg} / \mathrm{kg} 1 \% \mathrm{ketamine}$ and $0.5 \mathrm{mg} / \mathrm{kg} 1 \%$ propofol by the authors for sedoanalgesia. At five minute intervals the HR, SAP, DAP, MAP, $\mathrm{SpO}_{2}$ values and sedation scores were measured and recorded.

Results: Statistical evaluation found a statistically significant increase in SAP, DAP and MAP values measured at the $5^{\text {th }}$ minute in Group I patients compared to preoperative values. In Group I patients, when the heart rate measured in the 1 st and $5^{\text {th }}$ minutes are compared with preoperative values there was a statistically significant increase identified. In Group II patients, there was a statistically significant fall in $\mathrm{SpO}_{2}$ values in the 1st and 5th minutes compared with preoperative values. When patient and surgeon satisfaction are compared with Group I, Group II was found to be statistically significantly higher. Though the blood pressure and heart rate increases in Group I patients were statistically significant, they were not at levels that required clinical intervention and/or treatment. Similarly the $\mathrm{SpO}_{2}$ decrease observed in Group II patients did not fall below $90 \%$ in any patient in spite of being statistically significant and rose again without clinical intervention and/or treatment.

Conclusion: In this study we showed that sedoanalgesia administration with propofol and ketamine may be an alternative method for patients undergoing minor urology interventions that does not disrupt hemodynamic and respiratory stability, does not delay patient discharge, has low side effect incidence and has high patient and surgeon satisfaction.
\end{abstract}

\section{Introduction}

For minor surgical interventions in the urology clinics at our hospital, like diagnostic and check-up cystoscopy, double-j catheter insertion and removal and uretroscopy, local anesthesia with lidocaine gels is the chosen method. Though local anesthetic is chosen due to reasons such as number of patients, insufficient personnel, lack of time or insufficient beds, this method does not provide the majority of patients with sufficient anesthesia or sufficient patient satisfaction. Additionally, a large proportion of patients have additional cardiovascular and pulmonary diseases and advanced age, adding to the importance of ensuring hemodynamic and respiratory stability. Recent Effective sedation and analgesia during procedures not only provides relief of suffering, but also frequently facilitates the successful and timely completion of the procedure. However, any of the agents used for sedation and/or analgesia may result in adverse effects. The two medications used in our study, propofol and ketamine, have been used for many

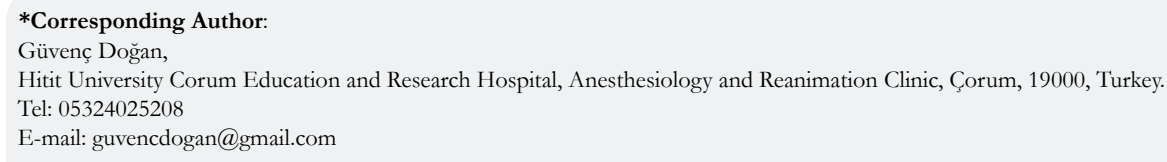

Citation: Kılıç I, Doğan G, Çakır E, Akdur F, Başkan S, et al., (2017) Sedoanalgesia Administration with Propofol and Ketamine for Minor Urologic Interventions. Int J Anesth Res. 5(3), 422-428. doi: http://dx.doi.org/10.19070/2332-2780-1700087

Copyright: Doğan $\mathbf{G}^{\circ}$ 2017. This is an open-access article distributed under the terms of the Creative Commons Attribution License, which permits unrestricted use, distri bution and reproduction in any medium, provided the original author and source are credited. 
years for anesthesia. It has been suggested that the combination of ketamine and propofol (K-P), or "ketofol" if the two agents are mixed in a single syringe, for procedural sedation allows for a reduction in the required dose of each individual agent, potentially mitigating the risk of adverse respiratory events compared to propofol alone or in combination with opioids [1-3].

We aimed to use both medications at subanesthetic doses to create sufficient sedation, analgesia and amnesia, minimize medication-linked side effects and ensure hemodynamic and respiratory stability.

\section{Material And Method}

Our study was completed after receiving consent from patients undergoing minor urologic interventions at the Urology Clinic of Ankara Numune Education and Research Hospital.

\section{Patient Choice}

Patients with planned minor urologic interventions were assessed in the ward one day before the operation. Fifty-three patients aged from 19 to 85 years, with physical situation ASA I-III risk group were included in the study after the protocol was explained and written consent obtained.

Patients with planned cauterization, with expected operation duration of longer than ten minutes, with communication problems (tongue problems, health, etc.), with known sensitivity or allergy to medications used or raw eggs, ASA IV and above, and those who did not accept sedation for the planned operation were excluded from the study.

\section{Study Protocol}

Patients were given no premedication and were taken for the operation after six hours of fasting. Patients brought into the operating room had electrocardiography (ECG), pulse oxymetry and oxygen saturation $\left(\mathrm{SpO}_{2}\right)$, and non-invasive blood pressure monitoring. Patients had a $22 \mathrm{G}$ intravenous cannula inserted into the dorsal left hand, and $5 \mathrm{ml} / \mathrm{min}$ isotonic sodium chloride infusion had begun. A nasal O2 cannula was inserted for use if needed.

Patients had heart rate (HR), systolic arterial pressure (SAP), diastolic arterial pressure (DAP), mean arterial pressure (MAP) and oxygen saturation $\left(\mathrm{SpO}_{2}\right)$ values measured. Sedation scores were measured with a 5 -point sedation scale (Table 1$)$. Basal values $(0$ min) were recorded.

Patients were randomly divided into two groups. Patients in Group I had local anesthesia administered by the surgeon using intraurethral lidocaine gel $\left(\right.$ Cathajel $\left.^{\circledR}\right)$ five minutes before the operation. Five minutes after the administration of local anesthetic gel, the operation began. Patients in Group II had $0.015 \mathrm{mg} / \mathrm{kg}$ midozolam (Dormicum) for premedication and were taken for operation. After premedication, $0.5 \mathrm{mg} / \mathrm{kg} 1 \%$ ketamine (Ketalar $\left.{ }^{\circledR}\right)$ and $0.5 \mathrm{mg} / \mathrm{kg} 1 \%$ propofol $\left(\right.$ Propofol ${ }^{\circledR}$ ) were administered intravenously for sedoanalgesia. After sedation was ensured, permission was given for the operation to begin.

In the first and fifth minutes of the operation, the HR, SAP, DAP,
$\mathrm{MAP}$ and $\mathrm{SpO}_{2}$ values and sedation scores of patients were measured and recorded.

After the operation, patients taken to the recovery room had HR, $\mathrm{SAP}, \mathrm{DAP}, \mathrm{MAP}$ and $\mathrm{SpO}_{2}$ values and sedation scores measured and recorded at five minute intervals.

Immediately after the operation the surgeon assessed satisfaction on a four-point scale (Table 4). One hour after the operation the patient assessed satisfaction on the same scale.

After the operation, in the $15^{\text {th }}$ minute, patients in the recovery room without sedation scores of 1 (fully awake and oriented) had $\mathrm{HR}$, SAP, DAP, MAP and $\mathrm{SpO}_{2}$ values and sedation scores recorded until sedation score reached 1 .

\section{Data Analysis}

Analysis of data was completed with the SPSS 15.0 statistical program. When evaluating data frequency distribution, mean, standard deviation, percentage and diagonal tables were used. For categorical comparisons the chi-square or Fisher Exact test were used. To determine any differences between the groups in the research, the Student $t$ test was used, while differences within the groups were determined with the repeated measurements variance analysis. For multiple comparisons, in situations with a difference between groups, the Tukey HSD and Dunnet tests were applied to determine which group caused the difference. Probability $(\mathrm{P})$ of 0.05 or less was accepted as significant and indicating a difference between groups, larger values were insignificant and indicated no difference between the groups.

\section{Results}

There was no statistically significant difference between the groups in terms of sex, weight and age $(p>0.05)$, there was a statistically significant difference between the groups in terms of ASA (American Society of Anesthesiologists) classification ( $\mathrm{p}<$ 0.05) (Table 1).

Comparisons between the groups found that systolic arterial pressure values measured at the start of the operation and the $15^{\text {th }}$ minute did not have a statistically significant difference $(p>0.05)$ (Table 2), while systolic arterial pressure values in the $1 \mathrm{st}$ and 5 th minutes were statistically significantly different $(\mathrm{p}<0.05)$.

In patients of Group I, the systolic arterial pressure values measured in the $1^{\text {st }}$ and $5^{\text {th }}$ minutes were statistically significantly higher than those measured in Group II (Table 3).

In Group I patients, SAP values in the $1^{\text {st }}$ and $15^{\text {th }}$ minutes were not statistically significantly different compared with preoperative values $(p>0.05)$, while SAP values from the $5^{\text {th }}$ minute were statistically significantly high compared to the preoperative values $(\mathrm{p}$ $<0.001)$.

In patients in Group II, when simultaneous SAP values were compared with preoperative values there was no statistically significant differences found $(\mathrm{p}<0.05)$ (Table 4).

For Group I patients, DAP values measured in the $1^{\text {st }}$ and $15^{\text {th }}$ 
Table 1. Demographic Characteristics (Mean \pm SD).

\begin{tabular}{|c|c|c|c|}
\hline & Group I (n= 30) & Group II (n = 23) & $\mathbf{P}$ \\
\hline Sex $(\mathrm{F} / \mathrm{M})$ & $10 / 20$ & $8 / 15$ & 0.912 \\
\hline Weight $(\mathrm{kg})$ & $73.40 \pm 10.68$ & $71.30 \pm 13.74$ & 0.535 \\
\hline Age $($ Year $)$ & $51.63 \pm 18.79$ & $51.96 \pm 15.27$ & 0.947 \\
\hline ASA I/II/III & $2 / 23 / 5$ & $3 / 9 / 11$ & 0.020 \\
\hline
\end{tabular}

Table 2. Comparison of Systolic Arterial Pressure between the Groups (Mean \pm SD).

\begin{tabular}{|c|c|c|c|}
\hline SAP & Group I $(\mathbf{n}=\mathbf{3 0})$ & Group II $(\mathbf{n}=\mathbf{2 3})$ & $\mathbf{P}$ \\
\hline Preop & $147.30 \pm 18.52$ & $147.22 \pm 15.19$ & 0.986 \\
\hline $1^{\text {st }}$ min. & $152.70 \pm 21.50$ & $138.83 \pm 19.86$ & 0.020 \\
\hline $5^{\text {th }}$ min. & $159.30 \pm 22.93$ & $145.30 \pm 19.74$ & 0.023 \\
\hline $15^{\text {th }}$ min. & $149.43 \pm 23.35$ & $145.39 \pm 21.25$ & 0.519 \\
\hline
\end{tabular}

Table 3. Comparison of Systolic Arterial Pressure within Groups (p).

\begin{tabular}{|c|c|c|c|}
\hline \multicolumn{2}{|c|}{ SAP } & Group I $(\mathbf{N}=\mathbf{3 0})$ & Group II $(\mathbf{n}=\mathbf{2 3})$ \\
\hline \multirow{3}{*}{ Preop } & $1^{\text {st }}$ min. & 0.082 & 0.058 \\
\cline { 2 - 4 } & $5^{\text {th }}$ min. & $0.000^{* * *}$ & 0.910 \\
\cline { 2 - 4 } & $15^{\text {th }}$ min. & 0.722 & 0.921 \\
\hline
\end{tabular}

$*$ Statistically significant difference compared to preop measurement $(\mathrm{p}<0.05)$.

** Statistically significant difference compared to preop measurement $(\mathrm{p}<0.01)$.

*** Statistically significant difference compared to preop measurement $(\mathrm{p}<0.001)$.

Table 4. Comparison of Diastolic Arterial Pressure Between Groups (Mean \pm SD).

\begin{tabular}{|c|c|c|c|}
\hline DAP & Group I $(\mathbf{n}=\mathbf{3 0})$ & Group II $(\mathbf{n}=\mathbf{2 3})$ & $\mathbf{p}$ \\
\hline Preop & $82.10 \pm 9.45$ & $85.30 \pm 7.32$ & 0.185 \\
\hline $1^{\text {st }}$ min. & $83.77 \pm 12.06$ & $84.04 \pm 12.43$ & 0.935 \\
\hline $5^{\text {th }}$ min. & $87.87 \pm 13.54$ & $87.48 \pm 13.21$ & 0.917 \\
\hline $15^{\text {th }}$ min. & $84.20 \pm 11.85$ & $85.61 \pm 13.36$ & 0.686 \\
\hline
\end{tabular}

minutes were not statistically significantly different from preoperative values $(\mathrm{p}>0.05)$, DAP values in the 5 th minute were statistically significantly high compared to preoperative values $(\mathrm{p}<$ 0.001) (Table 5).

For Group II patients, simultaneous DAP values were not found to be statistically significantly different when compared to preoperative values $(p>0.05)$ (Table 5).

Comparison of simultaneous mean arterial pressure values between the groups did not find any statistically significant difference between the groups $(\mathrm{p}>0.05)$ (Table 6$)$.

When MAP values for Group I patients are compared, values in the $1^{\text {st }}$ and $15^{\text {th }}$ minute were not statistically significantly different to preoperative values $(\mathrm{p}>0.05)$; values measured in the $5^{\text {th }}$ minute were statistically significantly higher compared to preoperative values $(\mathrm{p}<0.001)$ (Table 7$)$.

When MAP values for Group II patients are compared, there was no statistically significant difference found between simultaneous MAP values and preoperative values $(\mathrm{p}>0.05)$.
Comparison between the groups found no statistically significant difference in terms of simultaneous heart rate values $(p>0.05)$ (Table 8).

In terms of HR values for Group I patients, there was no statistically significant difference between values in the $15^{\text {th }}$ minute compared with preoperative values $(\mathrm{p}>0.05)$, while $\mathrm{HR}$ values measured in the $1^{\text {st }}(\mathrm{p}<0.05)$ and $5^{\text {th }}$ minute $(\mathrm{p}<0.001)$ were found to be statistically significantly high compared to preoperative values (Table 9).

In terms of HR values for Group II patients, there was no statistically significant difference found when simultaneous HR values are compared with preoperative values $(\mathrm{p}>0.05)$.

Comparison between the groups found no statistically significant difference between $\mathrm{SpO}_{2}$ values measured preoperatively, in the $5^{\text {th }}$ and $15^{\text {th }}$ minutes $(\mathrm{p}>0.05)$, while $\mathrm{SpO}_{2}$ values measured in the $1^{\text {st }}$ minute were statistically significantly different between the groups $(\mathrm{p}<0.05)$ (Table 10). 
Table 5. Comparison of Diastolic Arterial Pressure within Groups (p).

\begin{tabular}{|c|c|c|c|}
\hline \multicolumn{2}{|c|}{ DAP } & Group I (n= 30) & Group II $(\mathbf{n}=\mathbf{2 3})$ \\
\hline \multirow{4}{*}{ Preop } & $1^{\text {st }} \min$ & 0.570 & 0.862 \\
\cline { 2 - 4 } & $5^{\text {th }} \min$ & $0.001 * * *$ & 0.562 \\
\cline { 2 - 4 } & $15^{\text {th }} \min$ & 0.388 & 0.997 \\
\hline
\end{tabular}

* Statistically significant difference compared to preop measurement $(\mathrm{p}<0.05)$.

** Statistically significant difference compared to preop measurement $\mathrm{p}<0.01)$.

*** Statistically significant difference compared to preop measurement $(\mathrm{p}<0.001)$.

Table 6. Comparison of Mean Arterial Pressure between the Groups (Mean \pm SD).

\begin{tabular}{|c|c|c|c|}
\hline MAP & Group I (n = 30) & Group II (n = 23) & P \\
\hline Preop & $103.87 \pm 11.17$ & $105.83 \pm 9.02$ & 0.495 \\
\hline $1^{\text {st }} \min$ & $106.80 \pm 13.52$ & $102.26 \pm 14.26$ & 0.242 \\
\hline $5^{\text {th }} \min$ & $111.70 \pm 15.47$ & $106.65 \pm 14.37$ & 0.230 \\
\hline $15^{\text {th }} \min$ & $105.93 \pm 14.36$ & $105.57 \pm 15.09$ & 0.928 \\
\hline
\end{tabular}

Table 7. Comparison of Mean Arterial Pressure within Groups (p).

\begin{tabular}{|c|c|c|c|}
\hline \multicolumn{2}{|c|}{ MAP } & Group I $(\mathbf{n}=\mathbf{3 0})$ & Group II $(\mathbf{n}=\mathbf{2 3})$ \\
\hline \multirow{4}{*}{ Preop } & $1^{\text {st }} \min$ & 0.176 & 0.289 \\
\cline { 2 - 4 } & $5^{\text {th }} \min$ & $0.000 * * *$ & 0.970 \\
\cline { 2 - 4 } & $15^{\text {th }} \min$ & 0.438 & 0.999 \\
\hline
\end{tabular}

* Statistically significant difference compared to preop measurement $(\mathrm{p}<0.05)$.

** Statistically significant difference compared to preop measurement $(\mathrm{p}<0.01)$.

*** Statistically significant difference compared to preop measurement $(\mathrm{p}<0.001)$.

Table 8. Comparison of Heart Rate between the Groups (Mean \pm SD).

\begin{tabular}{|c|c|c|c|}
\hline HR & Group I $(\mathbf{n}=\mathbf{3 0})$ & Group II $(\mathbf{n}=\mathbf{2 3})$ & $\mathbf{P}$ \\
\hline Preop & $86.47 \pm 17.89$ & $87.26 \pm 13.59$ & 0.860 \\
\hline $1^{\text {st }} \min$ & $89.83 \pm 19.15$ & $86.00 \pm 12.90$ & 0.413 \\
\hline $5^{\text {th }} \min$ & $93.07 \pm 19.33$ & $86.26 \pm 13.47$ & 0.156 \\
\hline $15^{\text {th }} \min$ & $88.17 \pm 19.09$ & $84.13 \pm 13.41$ & 0.392 \\
\hline
\end{tabular}

Table 9. Comparison of Heart Rate within Groups (p).

\begin{tabular}{|c|c|c|c|}
\hline \multicolumn{2}{|c|}{ HR } & Group I (n= 30) & Group II $(\mathbf{n}=\mathbf{2 3})$ \\
\hline \multirow{4}{*}{ Preop } & $1^{\text {st }} \min$ & $0.039^{*}$ & 0.705 \\
\cline { 2 - 4 } & $5^{\text {th }} \min$ & 0.000 & 0.823 \\
\cline { 2 - 4 } & $15^{\text {th }} \min$ & 0.453 & 0.080 \\
\hline
\end{tabular}

* Statistically significant difference compared to preop measurement $(\mathrm{p}<0.05)$.

** Statistically significant difference compared to preop measurement $(\mathrm{p}<0.01)$.

*** Statistically significant difference compared to preop measurement $(\mathrm{p}<0.001)$.

Table 10. Comparison of $\mathrm{SpO}_{2}$ between the Groups (Mean $\pm \mathrm{SD}$ ).

\begin{tabular}{|c|c|c|c|}
\hline $\mathbf{S p O}_{2}$ & Group I (n = 30) & Group II (n = 23) & P \\
\hline Preop & $96.20 \pm 2.16$ & $96.09 \pm 1.83$ & 0.841 \\
\hline $1^{\text {st }}$ min. & $96.13 \pm 2.4$ & $93.91 \pm 2.50$ & 0.002 \\
\hline $5^{\text {th }}$ min & $96.20 \pm 2.38$ & $94.83 \pm 2.72$ & 0.056 \\
\hline $15^{\text {th }}$ min & $95.97 \pm 2.44$ & $95.96 \pm 2.01$ & 0.987 \\
\hline
\end{tabular}


When $\mathrm{SpO}_{2}$ values in Group I patients were measured in the $1^{\text {st }}$, $5^{\text {th }}$ and $15^{\text {th }}$ minute, there was no statistically significant difference compared to preoperative values $(p>0.05)$ (Table 11).

In Group II patients, $\mathrm{SpO}_{2}$ values measured in the $15^{\text {th }}$ minute were not statistically significantly different from preoperative values $(\mathrm{p}>0.05), \mathrm{SpO}_{2}$ values in the $1^{\text {st }}$ and $5^{\text {th }}$ minutes were found to be statistically low compared to preoperative values $(p<0.01)$ (Table 11).
Comparing the groups in terms of patient satisfaction, there was a statistically significant difference between the groups $(p<0.001)$. Accordingly, patients in Group II were more satisfied compared to patients in Group I (Table 12).

When groups are compared in terms of surgeon satisfaction, there was a statistically significant difference determined between the groups $(\mathrm{p}<0.001)$. Accordingly, surgeons operating on Group II patients were more satisfied compared to surgeons operating on Group I patients (Table 13).

Table 11. Comparison of $\mathrm{SpO}_{2}$ within Groups (p).

\begin{tabular}{|c|c|c|c|}
\hline \multicolumn{2}{|c|}{ SpO $_{2}$} & Group I $(\mathbf{n}=30)$ & Group II $(\mathbf{n}=\mathbf{2 3})$ \\
\hline \multirow{4}{*}{ Preop } & $1^{\text {st }} \min$ & 0.962 & $0.000^{* * *}$ \\
\cline { 2 - 4 } & $5^{\text {th }} \min$ & 1.000 & $0.002^{* *}$ \\
\cline { 2 - 4 } & $15^{\text {th }} \min$ & 0.392 & 0.969 \\
\hline
\end{tabular}

$*$ Statistically significant difference compared to preop measurement $(\mathrm{p}<0.05)$.

$* *$ Statistically significant difference compared to preop measurement $(\mathrm{p}<0.01)$.

$* * *$ Statistically significant difference compared to preop measurement $(p<0.001)$.

Table 12. Comparison of Patient Satisfaction between Groups (n/\%).

\begin{tabular}{|c|c|c|c|c|}
\hline \multicolumn{2}{|c|}{} & Group I (n = 30) & Group II (n = 23) & P \\
\hline \multirow{4}{*}{$\begin{array}{c}\text { Patient } \\
\text { Satisfaction }\end{array}$} & 0 & $15 / 50.0 \%$ & -- & \multirow{2}{*}{0.000} \\
\cline { 2 - 4 } & 1 & $12 / 40.0 \%$ & $2 / 8.70 \%$ & \\
\cline { 2 - 4 } & 2 & $3 / 10.0 \%$ & $14 / 60.87 \%$ & \\
\cline { 2 - 4 } & 3 & -- & $7 / 30.43 \%$ & \\
\hline
\end{tabular}

Table 13. Comparison of Surgeon Satisfaction between Groups (n/\%).

\begin{tabular}{|c|c|c|c|c|}
\hline \multicolumn{2}{|c|}{} & Group I (n= 30) & Group II (n = 23) & P \\
\hline \multirow{4}{*}{$\begin{array}{c}\text { Surgeon } \\
\text { Satisfaction }\end{array}$} & 0 & -- & -- & \\
\cline { 2 - 4 } & 1 & $20 / 66.7 \%$ & -- & \multirow{3}{*}{0.000} \\
\cline { 2 - 4 } & 2 & $10 / 33.3 \%$ & $14 / 60.87 \%$ & \\
\cline { 2 - 5 } & 3 & -- & $9 / 39.13 \%$ & \\
\hline
\end{tabular}

\section{Discussion}

In our study we administered low dose propofol and ketamine combined sedation and analgesia to patients with planned minor urologic interventions at Ankara Numune Education and Research Hospital Urology clinics. By combining propofol and ketamine to reduce unwanted side effects linked to both medications and not disrupt hemodynamic and respiratory stability, we aimed to ensure reliable, effective and rapid reversible sedation, analgesia and amnesia for patients undergoing the procedure.

Propofol and ketamine are reliable medications used in a variety of areas in anesthetic practice for many years, such as conscious sedation, anesthesia induction and maintenance. The uses of both medications alone at sedative doses have many wanted and unwanted effects. Propofol may be chosen for its rapid onset of effect, anti-emetic effect, antioxidant properties, ensuring amnesia, anticonvulsant effects and very rapid recovery. However, its use is limited by dose-linked increasing hypotension incidence, respiratory depression and suppression of the response to $\mathrm{CO}_{2}$ respira- tion. Ketamine is a very good analgesic, amnesic and sedative. Additionally it protects muscle tone, does not suppress protective respiratory tract reflexes and suppresses spontaneous respiration at lower rates. In spite of these beneficial effects, at increasing doses it may cause delayed recovery, increased incidence of nausea-vomiting, vision disorders (blurry vision, nystagmus) and psychomimetic reactions (dreams, delirium, hallucinations) which limits the use of this medication alone as a sedative [3, 4].

Some researchers have reported that the use of ketamine together with a sedative-hypnotic or general anesthetic causes a reduction in psychomimetic reactions $[4,5]$. In our study ketamine was combined with propofol; we reduced the ketamine dose necessary for the procedure and used ketamine with a general anesthetic. Thus, none of the psychomimetic reactions and vision disorders mentioned above was observed in any of our patients. One patient developed severe nausea in the recovery room, but did not vomit. This patient was treated with $4 \mathrm{mg}$ intravenous ondansetron. No patient had delayed discharge. 
Fridbeg et al., in a study of 1264 outpatient plastic surgery cases over five years, began with midazolam administration followed by propofol infusion and after hypnosis administered $50 \mathrm{mg}$ bolus ketamine. Later patients were administered local anesthetic injections and for nearly two hours until the end of the procedure propofol infusion continued. In this study the majority of patients had saturation above $90 \%$ in room air and the aim was to ensure deep sedation. Patients were not intubated and inhalation anesthesia was not used. In $95 \%$ of cases the total ketamine dose used was $200 \mathrm{mg}$ or less. None of the patients experienced hallucinations. The dreams due to ketamine were short and in this study they stated that the risky period was controlled by propofol [6].

In the ketamine waking period, there are negative changes in perception and assessment of visual and audio stimulations. These effects are reported to reduce with benzodiazepines [5]. The combination of the short-effect benzodiazepine midazolam with ketamine is reported to be successful in preventing these side effects [5]. A study by Nakagawa et al., found midazolam premedication reduced the amount of propofol necessary for sedation, increased the incidence of intraoperative amnesia and did not affect the incidence of other complications [7].

In light of this information, for our patients in Group II immediately before sedoanalgesia with ketamine and propofol we administered $0.015 \mathrm{mg} / \mathrm{kg}$ midazolam and no patient experienced hallucinations.

A double-blind study by Messenger et al., in the emergency service randomized 63 patients after monitoring and if necessary insertion of nasal oxygen cannula. One group was given $0.3 \mathrm{mg} /$ $\mathrm{kg}$ ketamine, while the other group was given $1.5 \mu / \mathrm{kg}$ fentanyl. Two minutes after ketamine or fentanyl, patients were given 0.4 $\mathrm{mg} / \mathrm{kg}$ propofol and $0.1 \mathrm{mg} / \mathrm{kg}$ propofol was given every $30 \mathrm{sec}-$ onds until sufficient sedation ensured. In the fentanyl group, there were higher rates of desaturation and requirements for oxygen support. In the ketamine group there was a higher requirement for propofol. In conclusion, though adding a dissociative dose of ketamine to propofol sedation led to requirements for a higher dose of propofol, it was found to be more reliable than fentanyl in terms of cardiorespiratory complications [8].

A study by Chiaretti et al., divided twenty acute lymphoblastic leukemia patients requiring lumbar puncture into two groups. One group was administered propofol-alfentanil, while the other group was administered propofol-ketamine sedoanalgesia. In spite of sufficient sedation and analgesia obtained with both protocols, the alfentanil group had higher rates of respiratory depression observed. Due to the lower incidence of side effects, the propofol-ketamine combination was found to be more reliable [9].

In our study patients in Group II were observed to have a statistically significant fall in saturation values measured 1 and 5 minutes after propofol and ketamine sedation. Despite the fact that this result was statistically significant, it was not clinically significant. None of our patients fell below $90 \%$ saturation while respiring room air or required oxygen support. We believe our use of low doses combining two medications allowed us to achieve these results.
While there is a temporary increase in cardiac index linked to ketamine dose, there is no difference observed in stroke index. With a slight direct myocardial depressant effect, ketamine causes an increase in peripheral vascular resistance and heart rate. These effects are linked to the presence of healthy sympathetic autonomous control and occur secondary to the effects of ketamine on the CNS [4, 5]. Propofol causes dose-linked hypotension due to reductions in cardiac output/cardiac index, beat volume and systemic vascular resistance.

A study by Hui et al., found significant differences between propofol, ketamine and propofol-ketamine combination groups in terms of arterial blood pressure. The use of the propofol and ketamine combination balanced the cardiostimulating effects of ketamine with the cardiodepressant effects of propofol and no changes in heart rate and arterial blood pressure were reported after painful stimulation [10].

In our study in Group II given propofol and ketamine sedoanalgesia, no patient was observed to have any statistically or clinically significant heart rate or arterial blood pressure changes. We consider that this is due to the balancing of the contrary effects on the cardiovascular system of the two medications, additionally this may be due to this sedoanalgesia method ensuring sufficient sedation and analgesia for patients during the procedure. As observed in the group with local anesthesia, we believe the statistically significant, but clinically not requiring treatment and/ or intervention, arterial blood pressure increase may be multifactorial. Among factors affecting the increase in blood pressure, being "aware" in the surgical environment, anxiety due to the procedure, discomfort due to position (especially for female patients), discomfort due to administration of local anesthetic gel, insufficient local anesthesia, feeling the procedure in spite of sufficient local anesthesia and previous hypertension disease may be listed.

Santiveri et al., in a double-blind study of 50 patients undergoing eye surgery with retrobulbar block randomized the patients into two groups. The first group was given sedation with $0.45 \mathrm{mg} / \mathrm{kg}$ propofol, while the second group was given $0.45 \mathrm{mg} / \mathrm{kg}$ propofol and $0.25 \mathrm{mg} / \mathrm{kg}$ ketamine. They found the addition of ketamine to the propofol administration for retrobulbar block provided better sedation without increasing the incidence of side effects [11].

A study of 114 patients undergoing painful orthopedic interventions in the emergency service by Willman and Andolfatto administered sedation with 1:1 ratio of ketamine and propofol from the same syringe. To complete the procedures the mean medication dose was $0.75 \mathrm{mg} / \mathrm{kg}$ propofol and $0.75 \mathrm{mg} / \mathrm{kg}$ ketamine $(0.2$ $\mathrm{mg} / \mathrm{kg}-2.05 \mathrm{mg} / \mathrm{kg}$ propofol and ketamine). They stated that the administration of ketamine and propofol sedation was a useful method due to low incidence of side effects, short recovery duration and high patient-personnel satisfaction [12].

A review by Blake investigated deep propofol and ketamine sedation supported by local anesthetic for 4800 patients undergoing cosmetic surgery operations from 1997 to 2007. They observed this was a technique with low complication rate, reliability, not requiring expensive equipment, providing postoperative analgesia and high patient and surgeon satisfaction [13].

In our study compared with the group with local anesthetic gel, 
patient and surgeon satisfaction was significantly higher for the the propofol and ketamine sedation group. Among the reasons for this may be development of amnesia in the patient, ease of completing the procedure due to their comfort during the procedure, shorter recovery times, lower complication rates and discharge of the patient on the same day.

In the urologic patient population, especially those with control cystoscopy for bladder tumors, the majority of patients are above the age of 60 and considering other accompanying diseases the necessity of ensuring hemodynamic and respiratory stability for these patients is clear. At the same time, in centers with a high amount of patients, being able to discharge the patient on the same day after these types of short procedure is a necessity. In our study we showed that sedoanalgesia with propofol and ketamine is an alternative method for these types of patients with rapid onset of effect, rapid recovery, low incidence of side effects, no lengthening of hospital stay, and with higher patient and surgeon satisfaction.

\section{Conclusion}

In this study we showed that sedoanalgesia administration with propofol and ketamine may be an alternative method for patients undergoing minor urologic interventions with rapid onset of effect that does not disrupt hemodynamic and respiratory stability, ensures rapid recovery, does not delaying discharge, has low incidence of side effects and higher patient and surgeon satisfaction.

\section{References}

[1]. Jalili M, Bahreini M, Doosti-Irani A, Masoomi R, Arbab M, et al., (2016) Ketamine-propofol combination (ketofol) vs propofol for procedural seda- tion and analgesia: systematic review and meta-analysis. Am J Emerg Med. 34(3): 558-69.

[2]. Victoria C Slavik, Peter J Zed (2007) Combination Ketamine and Propofol for Procedural Sedation and Analgesia. Pharmacotherapy. 27(11): 15881598.

[3]. Yan JW, McLeod SL, Iansavitchene A (2015) Ketamine-Propofol Versus Propofol Alone for Procedural Sedation in the Emergency Department: A Systematic Review and Meta-analysis. Acad Emerg Med. 22(9): 1003-13.

[4]. Tobias JD, Leder M (2011) Procedural sedation: A review of sedative agents, monitoring, and management of complications. Saudi J Anaesth. 5(4): 395410.

[5]. Deepa Kameswari Perumal, Mangaiarkkarasi Adhimoolam, Nitya Selvaraj, Suneeth Pullikotil Lazarus, Meher Ali Raja Mohammed (2015) Midazolam premedication for Ketamine-induced emergence phenomenon: A prospective observational study. J Res Pharm Pract. 4(2): 89-93.

[6]. Friedberg BL (1999) Propofol-ketamine technique: dissociative anesthesia for office surgery (a 5-year review of 1264 cases). Aesthetic Plast Surg. 23(1): 70-5.

[7]. Nakagawa M, Mammoto T, Hazama A, Kita T, Akamatsu T, et al., (2000) Midazolam premedication reduces propofol requirements for sedation during regional anesthesia. Can J Anaesth. 47(1): 47-9.

[8]. Messenger DW, Murray HE, Dungey PE, van Vlymen J, Sivilotti ML (2008) Subdissociative-dose ketamine versus fentanyl for analgesia during propofol procedural sedation: a randomized clinical trial. Acad Emerg Med. 15(10): 877-86.

[9]. Chiaretti A, Ruggiero A, Barone G, Antonelli A, Lazzareschi I, et al., (2010) Propofol/alfentanil and propofol/ketamine procedural sedation in children with acute lymphoblastic leukaemia: safety, efficacy and their correlation with pain neuromediator expression. Eur J Cancer Care (Engl). 19(2): 212 20.

[10]. Hui TW1, Short TG, Hong W, Suen T, Gin T, et al., (1995) Additive interactions between propofol and ketamine when used for anesthesia induction in female patients. Anesthesiology. 82(3): 641-8.

[11]. Santiveri X1, Moltò L, Rodríguez C, Sandín F, Vilaplana J, et al., (2006) Sedation and analgesia with propofol plus low-dose ketamine for retrobulbar block. Rev Esp Anestesiol Reanim. 53(9): 545-9.

[12]. Willman EV, Andolfatto G (2007) A prospective evaluation of "ketofol" ketamine/propofol combination) for procedural sedation and analgesia in the emergency department. Ann Emerg Med. 49(1): 23-30.

[13]. Blake DR (2008) Office-based anesthesia: dispelling common myths. Aesthet Surg J. 28(5): 564-70. 